\title{
Fission of highly excited nuclei investigated in complete kinematic measurements
}

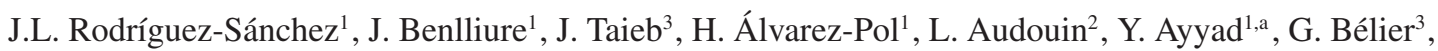 \\ G. Boutoux ${ }^{3}$, E. Casarejos ${ }^{4}$, A. Chatillon ${ }^{3}$, D. Cortina-Gil ${ }^{1}$, T. Gorbinet ${ }^{3}$, A. Heinz ${ }^{5}$, A. Kelić-Heil ${ }^{6}$, \\ N. Kurz ${ }^{6}$, B. Laurent ${ }^{3}$, J.-F. Martin ${ }^{3}$, C. Paradela ${ }^{1}$, E. Pellereau ${ }^{3}$, B. Pietras ${ }^{1}$, A. Prochazka ${ }^{6}$, D. Ramos $^{1}$, \\ C. Rodríguez-Tajes ${ }^{7}$, D. Rossi ${ }^{6}$, H. Simon ${ }^{6}$, L. Tassan-Got ${ }^{2}$, J. Vargas ${ }^{1}$, and B. Voss ${ }^{6}$ \\ ${ }^{1}$ Universidad de Santiago de Compostela, 15782 Santiago de Compostela, Spain \\ ${ }^{2}$ Institut de Physique Nucléaire d'Orsay, 91406 Orsay, France \\ ${ }^{3}$ CEA DAM, 91297 Arpajon, France \\ ${ }^{4}$ Universidad de Vigo, 36200 Vigo, Spain \\ ${ }^{5}$ Chalmers University of Technology, 41296 Gothenburg, Sweden \\ ${ }^{6}$ GSI Helmholtzzentrum für Schwerionenforschung GmbH, 64291 Darmstadt, Germany \\ ${ }^{7}$ Grand Accélérateur National d'Ions Lourds, 14076 Caen, France
}

\begin{abstract}
Fission is an extremely complex mechanism that requires a dynamical approach to describe the evolution of the process in terms of intrinsic and collective excitations of the nuclear constituents. In order to determine these effects a complex experimental setup was mounted at GSI, which allowed us for the first time the full identification in charge and mass of all fission fragments thanks to a magnetic separation and the use of the inverse kinematic technique. Moreover, we also measured the neutron multiplicities and the light-charged particles emitted in coincidence with fission. These complete kinematic measurements will be used to define sensitive observables to dissipative and transient effects in fission. In this manuscript we present the first results for the total fission cross sections.
\end{abstract}

\section{Introduction}

In 1940 the first description of the nuclear fission was proposed by Bohr and Wheeler [1] as a purely statistical model. At the same time and with the same purpose, Kramers [2] proposed a dynamical description of the fission process based on the coupling between internal and collective degrees of freedom through a dissipation parameter $(\beta)$ which reduces the fission decay width respect to the statistical model. Both descriptions were under discussion during four decades, however, in the 80's different measurements of pre-scission neutron multiplicities [3] provided values much larger than expected from the predictions using the Bohr-Wheeler's statistical model, which indicated the existence

\footnotetext{
a Present address: Research Center for Nuclear Physics, Osaka University, Ibaraki, Osaka 567-0047, Japan
}

This is an Open Access article distributed under the terms of the Creative Commons Attribution License 2.0, which permits unrestricted use, distribution, and reproduction in any medium, provided the original work is properly cited. 


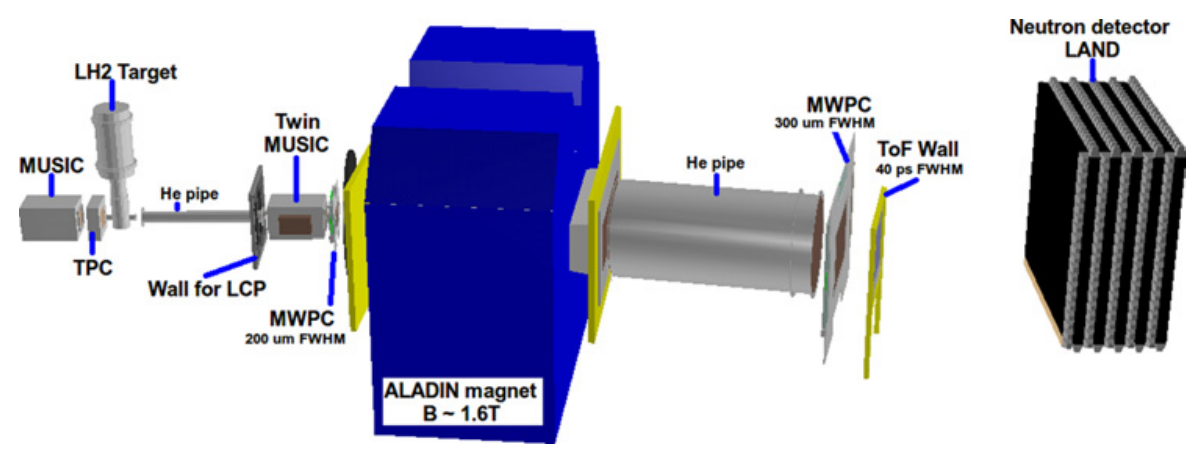

Figure 1. Schematic drawing of the experimental set-up used to investigate the reaction ${ }^{208} \mathrm{~Pb}+\mathrm{p}$ in inverse kinematics.

of a dissipative effect in the fission. The introduction of the dissipation implies that the system needs time to poputate the phase space and reach the equilibrium. Under this idea, Grangé [4] investigated the dependence of the fission decay width with the time solving the Fokker-Planck equation (FPE). The solution showed that the fission is highly suppressed at the onset of the process, requiring a certain transient time to reach the stationary fission rate given by Kramers. These dynamical effects appear on fissioning systems with high excitation energy, low angular momentum and near the ground-state deformation [4]. For this reason, we investigated the reaction ${ }^{208} \mathrm{~Pb}$ impinging onto a liquid hydrogen target at energies between 370 and $650 \mathrm{~A} \mathrm{MeV}$. Moreover the use of the inverse kinematic technique allowed us to measure all particles emitted in coincidence with the fission fragments providing a complete access.

\section{Experiment}

The fission setup situated at the Cave C at GSI is shown in Figure 1 where a small plastic-scintillator $\left(50 \times 30 \mathrm{~mm}^{2}\right)$ was used as trigger of the data acquisition. The characterization of the beam is made with an ionization chamber (MUSIC) [5], that provides the energy loss with high-resolution, and a Time Projection Chamber (TPC) [6], which provides the horizontal X and vertical Y position of the ions before the target with a resolution of $300 \mu \mathrm{m}$ (FWHM). A cylindrical target $(11.24 \mathrm{~mm}$ long and $30 \mathrm{~mm}$ diameter) was filled with liquid-hydrogen which was isolated by two windows consisting of aluminizedmylar foils of $35 \mu \mathrm{m}$. After the target, the identification of the fission fragments was made with a double ionization chamber (Twin MUSIC) (active volume of $50 \mathrm{~cm}$ long, $20 \mathrm{~cm}$ high and $20 \mathrm{~cm}$ wide) [7], which measures the nuclear charge and the X coordinate of both fission fragments with a resolution of $50 \mu \mathrm{m}$ (FWHM) and two multi-wire proportional chambers (MWPC), that provides the X and Y coordinates of the fission fragments with a resolution (FWHM) of $200 \mu \mathrm{m}$ and $1 \mathrm{~mm}$ [8], respectively. These position measurements allowed us to reconstruct the path-length and the $\mathrm{B} \rho$ of both fission fragments. Moreover, a ToF Wall [9] composed of 28 plastic-scintillator was placed behind the second MWPC to measure the time-of-flight with a resolution around $40 \mathrm{ps}$ (FWHM). In addition, two pipes filled with helium gas were mounted between the target and the double ionization chamber and between the magnet and second MWPC to reduce the energy and angular straggling of the fission fragments. To detect the light-charged particles in coincidence with the fission a segmented plastic-scintillator wall was placed around the first He pipe in front of the double ionization chamber. Finally, the neutron detector LAND [10] (1 m long, $2 \mathrm{~m}$ high and $2 \mathrm{~m}$ wide) was used to determine the neutron multiplicities. 


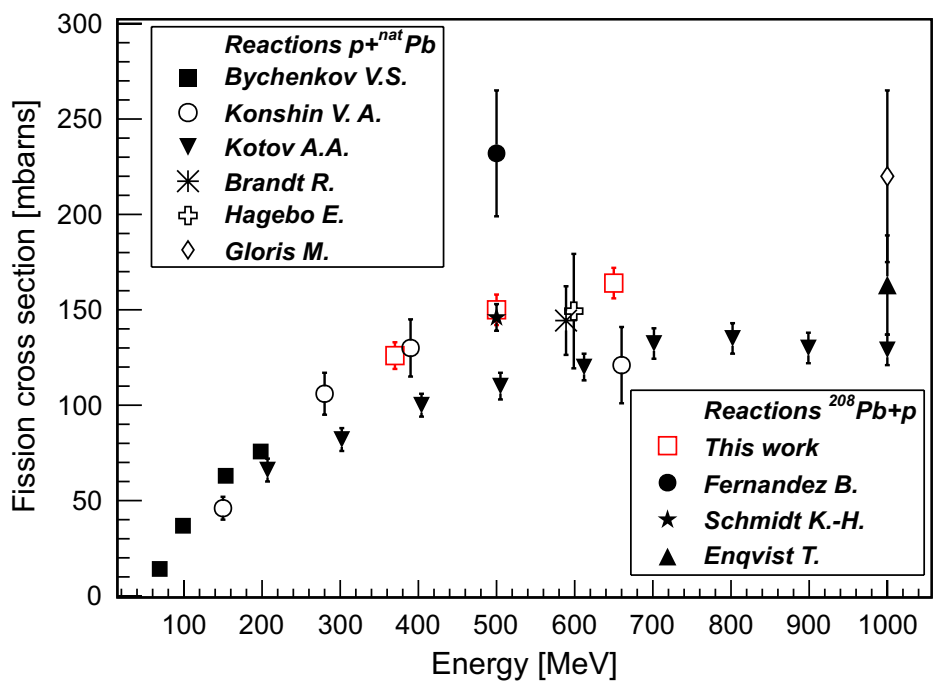

Figure 2. Total fission cross sections measured in the present work in comparison to previously measured data of the reactions ${ }^{208} \mathrm{~Pb}+\mathrm{p}$ and $\mathrm{p}+{ }^{\text {nat }} \mathrm{Pb}$.

\section{Results}

The total fission cross sections for proton-induced fission of ${ }^{208} \mathrm{~Pb}$ were measured at 370,500 and $650 \mathrm{~A} \mathrm{MeV}$. Measurements were performed with a hydrogen target of $(86 \pm 1) \mathrm{mg} / \mathrm{cm}^{2}$. The number of projectiles was determined from the reaction trigger provided by the small plastic-scintillator and the MUSIC while the number of fission events was determined using the double ionization chamber. Their statistical uncertainties given by Poisson statistics according to the observed number of counts are below $1 \%$ and are negligible in the fission cross-section uncertainties. The main contributions to the uncertainties are attributable to uncertainties of the target thickness, attenuation of the beam intensity in the target, fission events lost on the pipe and the detection efficiency of the TWIN chamber. The largest corrections are attributable to the attenuation of the beam intensity in the target considering the attenuation to half the target (values obtained for the energies 370,500 and $650 \mathrm{MeV}$ were $3.6 \%$, $3.8 \%$ and $4.0 \%$, respectively.), the correction of the fission events lost on the pipe and the detection efficiency of the TWIN chamber (around 88\%). The secondary reactions were also calculated with the nuclear reaction rate in half the target obtaining values smaller than $2.2 \%$ for all energies. Moreover, the fission fragments could undergo nuclear reactions between the target and the TWIN chamber and not be counted as fission events. This corresponding probability was calculated with the nuclear reaction rate of the different layers of matter, obtaining values below $0.5 \%$ for three energies.

Our results are shown in Figure 2 (open squares). In this figure we also show similar data found in the literature for the reactions $\mathrm{p}+{ }^{\text {nat }} \mathrm{Pb}$ and ${ }^{208} \mathrm{~Pb}+\mathrm{p}$ at different energies. As can be seen in the figure, our data have in general better accuracy than any of the previous ones. They seem consistent with the measurements between 50 and $200 \mathrm{MeV}$, which were performed by V.S. Bychenkov [11] (full squares), if one looks at the downward trend and also with measurement performed by T. Enqvist [12] (full triangle up) at the FRS if one looks at the upward trend. One can also see a discrepancy at $1 \mathrm{GeV}$ between the measurements performed by T. Enqvist [12] (full triangle up) and M. Gloris [13] (open diamond) but our results are only consistent with the Enqvist's measurement. Moreover, our results are in agreement with the values reported by E. Hagebo [14] (open cross) and R. Brandt [15] (star) who measured in direct kinematics the reaction $\mathrm{p}+{ }^{\text {nat }} \mathrm{Pb}$ at 600 and $590 \mathrm{MeV}$, respectively. However, 
the cross section obtained by B. Fernández [16] (full circle) at the FRS has a non-negligible deviation with respect to our results, which can be due to the limited acceptance of the spectrometer requiring large corrections. Also, the measurements performed by A.A. Kotov [17] (full triangles down) have a deviation, but these were performed in direct kinematics with a proton beam impinging on $\mathrm{a}^{\text {nat }} \mathrm{Pb}$ target, which could prevent fission fragments to escape from the target reducing the total fission cross section if one does not apply the accurate corrections. However, these measurements confirm the upward trend of our results. On the other hand our value of fission cross section at $500 \mathrm{MeV}$ is in agreement with the measurement performed by K.-H. Schmidt [18] (full star) in a similar setup at GSI. Moreover the fission cross section at $370 \mathrm{MeV}$ is in agreement with the results performed by V.A. Konshin [19] (open circles).

\section{Summary and conclusions}

In this work we have presented the first results for proton induced fission of ${ }^{208} \mathrm{~Pb}$ in inverse kinematics at 370, 500 and $650 \mathrm{~A} \mathrm{MeV}$. The combination of the inverse kinematic technique with a highly efficient detection set-up made it possible to obtain the total fission cross sections with high accuracy. The new data have made possible to clarify the previous controversial results obtained in different experiments.

\section{References}

[1] N. Bohr and J.A. Wheeler, Phys. Rev. 56, 426 (1939)

[2] H.A. Kramers, Physika VII 4, 284 (1940)

[3] A. Gavron et al., Phys. Rev. Lett. 48, 835 (1982)

[4] P. Grangé, L. Jun-Qing and H. A. Weidenmüller, Phys. Rev. C 27, 2063 (1983)

[5] M. Pfüzner et al., Nucl. Instr. Methods B 86, 213 (1994)

[6] R. Janik, A. Prochazka et al., Nucl. Instrum. Methods A 640, 54-57 (2011)

[7] B. Voss, "A low-mass Multi-Sampling Ionization chamber for fission studies", Nuclear Science Symposium and Medical Imaging Conference (NSS/MIC), 1767-1770 (2011)

[8] L. Audouin, Private communication

[9] A. Bail, "Time of flight measurement on the SOFIA experiment", Advancements in Nuclear Instrumentation Measurement Methods and their Applications (ANIMMA), 1-4 (2011)

[10] Th. Blaich et al., NIM A 314, 136-154 (1992)

[11] V.S. Bychenkov et al., Soviet Journal of Nuclear Physics, Vol. 17, 496 (1973)

[12] T. Enqvist et al. Nucl. Phys. A. 686, 481 (2001)

[13] M. Gloris, et al., Nucl. Inst. Meth. A 463593 (2001)

[14] E. Hagebo and T. Lund. J. inorg. nucl. Chem. 371569 (1975)

[15] R. Brandt et al. CERN 71, 2 (1971)

[16] B. Fernández-Dominguez et al. Nucl. Phys. A 747, 227 (2005)

[17] A.A. Kotov et al. Phys. Rev. C 74, 034605 (2006)

[18] K.-H. Schmidt et al. Phys. Rev. C 87, 034601 (2013)

[19] V.A. Konshin et al., Yadernaya Fizika Vol. 2, 682 (1965) 